\title{
Evaluación de la calidad de vida de adultos con discapacidad intelectual institucionalizados en Chile*
}

\section{Quality of Life Assessment of Institutionalized Adults with Intellectual Disabilities in Chile}

Recibido: octubre 24 de 2011| Revisado: mayo 3 de 2012 | Aceptado: agosto 15 de 2012

\author{
VANESSA Vega CóRdova** \\ Pontificia Universidad Católica de Valparaíso, Chile \\ CRISTINA JENARO RÍO *** \\ NOELIA FloRES ROBAINA \\ Universidad de Salamanca, España \\ MARIBel CRUZ ORTIZ***** \\ Universidad Autónoma de San Luis Potosí, México \\ ANDRÉS LERDO DE TEJADA ****** \\ Fundación Coanil, Chile
}

doi:10.11144/Javeriana.UPSY12-2.ecva

Para citar este artículo: Vega, V., Jenaro, C., Flores, N., Cruz, M. \& Lerdo de Tejada, A. (2013). Evaluación de la calidad de vida de adultos con discapacidad intelectual institucionalizados en Chile. Universitas Psychologica, 12(2), 471-481.

* Agradecemos a la Fundación Coanil por haber autorizado el desarrollo de la presente investigación en sus Centros y a todos los profesionales y personas con discapacidad que han permitido el buen término de este proyecto.

** Becaria CONICYT. Profesora Escuela de Pedagogía de la Pontificia Universidad Católica de Valparaíso (Chile). Profesora especialista en educación especial de personas con discapacidad intelectual y audición y lenguaje. Escuela de Pedagogía Pontificia Universidad Católica de Valparaíso, Avda. El Bosque 1290-Santa Inés. Viña del Mar (Chile). Teléfono: (56) (32) 2274398. Fax: (56) (32) 2274359. E-mail: vanejuay@gmail. com

**** Facultad de Psicología. Universidad de Salamanca España.E-mails: crisje@usal.es,nrobaina@usal. es

****** Universidad Autónoma de San Luis Potosí, México.E-mail: redazul@hotmail.com

******* Director Área Protección y Familia, Fundación Coanil Chile.E-mail: andresldt@coanil.cl
RESUMEN

El presente estudio analiza la calidad de vida de una muestra de 285 adultos institucionalizados con discapacidad intelectual. La calidad de vida, evaluada a través de profesionales, se concibe desde el modelo de ocho dimensiones de Schalock y Verdugo. Se ofrecen además evidencias de la adecuación (fiabilidad y validez) de la Escala Transcultural de Indicadores de Calidad de Vida para su aplicación con esta población. Los resultados revelaron diferencias significativas en importancia y uso de las diferentes dimensiones de calidad de vida, así como sobre sus valoraciones en función del grado de discapacidad de los participantes. La duración de la institucionalización tuvo un impacto significativo en las valoraciones de la importancia de las distintas dimensiones de calidad de vida. Se incluye una discusión sobre las percepciones de los proveedores de servicios acerca de la calidad de vida de sus usuarios y del impacto de la institucionalización.

Palabras clave autores

Calidad de vida, discapacidad intelectual, institucionalización.

Palabras clave descriptores

Investigación cuantitativa, Psicología del desarrollo, Envejecimiento.

\section{A B S T R A C T}

This study analyzes the quality of life for a sample of 285 institutionalized adults with intellectual disabilities. Quality of life, as assessed by professionals, is conceptualized according to the eight domains model from Schalock and Verdugo. The study also offers evidence of the adequacy (reliability and validity) of the Cross-cultural Measure of Quality of Life Indicators for use with this population. The results revealed significant differences in importance and use of the different dimensions of quality of life, and evaluation ratings of quality of life, based on the degree of disability of the participants. Length of institutionalization significantly impacted on ratings of importance of the different domains of quality of life. Discussion on further research on perceptions of service providers concerning the quality of life of its users and the impact of institutionalization is included.

Key words authors

Quality Of Life, Intellectual Disabilities, Institutionalization.

Key words plus

Quantitative Research, Development Psychology, Elderly. 
El constructo de calidad de vida ha generado y suscita en la actualidad un gran interés. Desde un punto de vista centrado en el individuo, es posible distinguir dos modelos fundamentales. Uno, centrado en la salud y característico de un enfoque médico, y otro centrado en el bienestar más general, característico de un enfoque psicosocial. En ambos casos, la meta última es mejorar las condiciones de vida de la población (Cummins, 2005). Desde un modelo médico-clínico, el concepto utilizado suele ser el de calidad de vida relacionada con la salud, conocido por sus siglas en inglés como HLQoL. Desde dicho marco se acentúan las condiciones de salud y su impacto en el bienestar general, así como el impacto de la actuación médico-sanitaria en la calidad de vida asociada a la salud (p. ej., Albert, 1997; Anderson, Aaronson \& Wilkin, 1993; Garin et al., 2009; Murdaugh, 1997; Naughton \& Wiklund, 1993; Palfreyman, Tod, Brazier \& Michaels, 2010; Quittner, Cruz, Modi \& Marciel, 2009; Revicki \& Kaplan, 1993; Solans et al., 2008). Es por tanto un modelo de especial relevancia para poblaciones con problemas de salud crónica: para pacientes y para actuaciones centradas en mantener, conservar o, en forma óptima, reducir los síntomas y mejorar la salud.

Por su parte, desde un modelo psicosocial próximo a la economía, la sociología y la educación, el interés se centra en personas en situación de desventaja debido a la presencia de una discapacidad u otra condición (género, etnia, etc.). Desde este enfoque son especialmente relevantes los grupos de investigación centrados en la discapacidad in- telectual (Gomez, Verdugo \& Arias, 2010; Schalock, Bonham \& Verdugo, 2008). En este ámbito, el constructo de calidad de vida ha influenciado de manera positiva diferentes áreas, especialmente en cuanto a los servicios que se ofrecen a las personas que presentan discapacidad intelectual o del desarrollo. Este concepto proporciona una estructura sistemática para aplicar políticas y prácticas, esto es, servicios orientados a mejorar la calidad de vida (Schalock \& Verdugo, 2003; American Association on Intellectual and Developmental Disabilities [AAIDD], 2010).

Numerosos autores (Brown, 1988; Felce \& Perry, 1995; Schalock \& Verdugo, 2003; Schalock, Keith, Verdugo \& Gomez, 2010) han estudiado la calidad de vida desde diferentes perspectivas, tanto teóricas como empíricas, teniendo en cuenta tanto aspectos objetivos como subjetivos, entendiendo la calidad de vida como un concepto global, holístico y que no se centra en un único momento temporal. Para el presente estudio, se asumió el modelo de calidad de vida propuesto por Schalock y Verdugo (2003). Este modelo permite comprender mejor cómo ofrecer los apoyos necesarios para mejorar la calidad de vida. Se compone de ocho dimensiones y 24 indicadores (Tabla 1).

Dicho modelo fue la base para la construcción de la Escala Transcultural de Calidad de Vida. Numerosas investigaciones avalan las adecuadas propiedades psicométricas de la misma, así como su validez transcultural (Jenaro et al., 2005; Schalock, Gardner \& Bradley, 2007; Schalock \& Verdugo, 2003; Schalock et al., 2005; Vega, Jenaro, Cruz

\section{TABLA 1}

Dimensiones e indicadores de calidad de vida

\begin{tabular}{ll}
\hline \multicolumn{1}{c}{ Dimensiones } & \multicolumn{1}{c}{ Indicadores } \\
\hline Bienestar Emocional & Alegría, autoconcepto, ausencia de estrés. \\
Relaciones Interpersonales & Interacciones, relaciones de amistad, apoyos. \\
Bienestar Material & Estado financiero, empleo, vivienda. \\
Desarrollo Personal & Educación, competencia personal, realización. \\
Bienestar Físico & Atención sanitaria, estado de salud, actividades de la vida diaria, ocio. \\
Autodeterminación & Autonomía/control personal, metas y valores personales, elecciones. \\
Inclusión Social & Integración y participación en la comunidad, roles comunitarios, apoyos sociales. \\
Derechos & Legales, humanos (dignidad y respeto). \\
\hline
\end{tabular}

Fuente: Schalock y Verdugo (2003). 
\& Flores, 2011). Sin embargo, no existe evidencia empírica de su utilización en población chilena o en participantes con discapacidad intelectual institucionalizados, como es el caso que nos ocupa.

Desde hace más de una década, varios autores han tratado de analizar la relación entre servicios residenciales y calidad de vida (Brown \& Brown, 2005; Hatton, Emerson, Robertson, Henderson \& Cooper, 1995; Keith \& Rawlinson, 1998; Stancliffe, 2002; Young, 2006). Por ejemplo, Young (2006) pone de manifiesto cómo las alternativas residenciales en la comunidad son una ventaja frente a las oportunidades disponibles en las grandes instituciones residenciales. Y esto porque, entre otras variables, la vida en ambientes restrictivos puede influir en la autodeterminación y toma libre de decisiones (Stancliffe \& Parmenter, 1999). Por otro lado, en estas instituciones la calidad de vida experimentada por las personas con discapacidad viene determinada, en gran medida, por las políticas de la organización y los mecanismos de prestación de servicios (Keith \& Rawlinson, 1998).

Así pues, cada vez más las alternativas residenciales son objeto de análisis para determinar qué variables hacen de una residencia un verdadero hogar (Annison, 2000). Factores como la edad, cantidad de años de permanencia en la institución o los niveles de comportamiento adaptado o inadaptado se han encontrado asociados a la calidad de vida (Felce \& Emerson, 2001; Stancliffe, Hayden, Larson \& Lakin, 2002). En la misma línea, diferentes estudios han constatado que participar en actividades de empleo mejora la calidad de vida de las personas con discapacidad intelectual (Goode, 1989; Murphy, Rogan, Handley, Kincaid \& RoyceDavis, 2002).

En un intento por mejorar nuestra comprensión sobre el concepto de calidad de vida y el impacto de los servicios en dicha calidad, el presente estudio trató de responder a las preguntas: iexperimentan las personas con discapacidad intelectual institucionalizadas una vida de calidad?, iqué importancia y en qué grado se emplean las diferentes dimensiones de calidad de vida con estos residentes? Concretamente, se plantearon dos objetivos principales: 1) recoger evidencias adicionales sobre la adecu- ación de la Escala Trascultural de Calidad de Vida en adultos de Chile con discapacidad intelectual o del desarrollo institucionalizados; 2) describir la calidad de vida (importancia, utilización, valoración) de adultos chilenos con discapacidad intelectual que viven permanentemente en residencias.

En el estudio se plantean tres hipótesis que, a juicio de los profesionales, establecen que: 1) existirán diferencias significativas entre los juicios sobre importancia, utilización y valoración de calidad de vida; 2) la severidad de la discapacidad afectará a la calidad de vida (relación inversa, y significativa) y 3) los años de institucionalización afectarán significativamente a la calidad de vida (relación inversa y significativa).

\section{Método}

\section{Instrumento}

Como ya se mencionó, para medir la calidad de vida se utilizó la Escala Transcultural de Indicadores de Calidad de Vida (Jenaro et al., 2005; Verdugo, Schalock, Wehmeyer, Caballo \& Jenaro, 2001) que tiene por finalidad determinar la importancia, utilización y calidad percibida, que el profesional o el servicio atribuye a los indicadores fundamentales asociados a cada una de las ocho áreas de calidad de vida.

La escala está dividida en varios apartados. La primera parte contiene preguntas sobre información de la persona que responde y del usuario evaluado. La segunda parte incluye una descripción general del objetivo de la escala e instrucciones para que sea completada. En la tercera parte se describen las ocho dimensiones de calidad de vida. En la cuarta parte del instrumento, o parte central de la escala, aparecen los indicadores de las distintas dimensiones.

En la escala original cada indicador debe ser valorado de acuerdo a dos tipos de juicios: 1) valoración de la importancia que cada indicador tiene, a juicio de los evaluadores, en los usuarios con quienes trabaja y 2) utilización o grado (cuánto) en que cada indicador se utiliza o se trata de potenciar en los servicios que como profesional ofrece y apoyos 
que proporciona a adultos con discapacidad intelectual. A estos dos tipos de juicios, para la presente investigación, se añadió un apartado adicional en el que se solicita valorar la calidad de vida que el profesional considera que tiene el usuario objeto de evaluación (grado en que los diferentes indicadores de calidad de vida son experimentados por el usuario). Podría entenderse como la calidad de vida normativa, utilizando la terminología de Thompson et al. (2009), en el constructo objeto de interés. Estos tres aspectos se valoran en una escala de cuatro puntos, siendo 1 la mínima importancia, utilización o calidad de vida y 4 la máxima puntuación.

Para comprobar la adecuación de la escala se llevaron a cabo análisis de su fiabilidad y validez. En cuanto a la fiabilidad, el estadístico alfa de Cronbach fue superior a 0.9 para todas las dimensiones y versiones del instrumento, por lo que se pueden considerar plenamente satisfactorias. Por lo que se refiere al análisis de la fiabilidad de la escala mediante el método de división en mitades, se obtuvieron valoraciones superiores a 0.89 y correlaciones entre los ítems que oscilan de 0.67 a 0.76 , lo que ofrece apoyo a la fiabilidad de la escala.

En cuanto a la validez de la escala se consideraron los siguientes tipos de validez: 1) validez de constructo, mediante: a) análisis de los pesos factoriales de los ítems de importancia, uso y valoración, tras haber realizado un análisis factorial exploratorio; b) cálculo de las intercorrelaciones de las subescalas y c) relación de las puntuaciones en la escala de valoración de la calidad de vida con la severidad de la discapacidad -se espera una relación inversa-;2) validez discriminante, estableciendo el poder discriminatorio de las puntuaciones obtenidas en las subescalas de la valoración de calidad de vida, sobre los participantes que asisten o no a un taller laboral. Los resultados destacan que los pesos factoriales de los ítems fueron elevados y asociados a las escalas a las que pertenecen. Se obtuvieron además correlaciones moderadas-altas entre escalas y más altas entre escalas-total. Ello ofrece apoyo a la validez de constructo de la escala, en sus tres versiones.

\section{Participantes}

El estudio incluye información de un total de 285 personas institucionalizadas con discapacidad intelectual, mayores de 18 años, que pertenecen a los centros de la Fundación Coanil de las regiones Quinta y Metropolitana de Chile. La muestra fue de conveniencia, con una edad promedio de 29.3 años y un rango entre 18 y 51 años. El $49.5 \%$ eran hombres y el $50.5 \%$ mujeres. Según el grado de discapacidad, la mayoría de la muestra (55.4\%) presentaba una discapacidad severa, un $17.9 \%$, discapacidad moderada y un $16.5 \%$, discapacidad profunda, según criterios DSM-IV.

La media de años en la institución era de 14.4 años, con un rango entre menos de un año y 32 años. Un $22.1 \%$ de los participantes llevaba 23 años institucionalizado. Otro dato a destacar es que el $58.6 \%$ de la muestra no asiste a taller laboral u otro similar, a pesar de ser mayores de 18 años.

Las escalas fueron respondidas por 83 informantes. Para cada caso, se eligió a aquel profesional con mayor conocimiento del usuario en diversos contextos; cada uno de ellos contestó más de una entrevista sobre la calidad de vida de las personas con discapacidad intelectual. De dichos informantes $21.1 \%$ eran trabajadoras sociales, $22.5 \%$, profesores de educación especial y $25.5 \%$, personal de apoyo directo. Por término medio, los profesionales conocían a los usuarios desde hacía 7.81 años $(D E=8.53)$, con un rango entre 1 y 31 años.

\section{Procedimiento}

La investigación adoptó un diseño ex post facto que pretende evaluar la calidad de vida de las personas con discapacidad intelectual adultas, bajo el modelo de ocho dimensiones de calidad de vida de Schalock y Verdugo (2003). Se desarrolló en siete centros pertenecientes a la Fundación Coanil, entidad privada que desde 1974 viene trabajando en el área de la discapacidad y que es además la organización de servicios residenciales más grande de Chile.

La investigación siguió un proceso de tres fases. La primera supuso el contacto con directivos de la Fundación Coanil quienes aprobaron el protocolo 
de evaluación y con quienes se firmó un convenio de colaboración. La segunda consistió en la realización de charlas informativas a los diferentes funcionarios de la Fundación con el objetivo de que conocieran la investigación y se programara el trabajo con cada uno de los hogares. La tercera fase implicó la recolección de datos, que se llevó a cabo a través de entrevistas personales a profesionales o cuidadoras que trabajan en los hogares. La recolección de datos en siete hogares de la Fundación se ejecutó durante los meses de agosto a diciembre de 2008. Las entrevistas fueron realizadas por la responsable principal del estudio, junto con alumnos de quinto año de la carrera de Educación Diferencial de la Pontificia Universidad Católica de Valparaíso. Los informantes de los diferentes centros residenciales consintieron en participar tras garantizárseles la confidencialidad de la información recogida.

\section{Análisis estadísticos}

Las pruebas para el contraste de hipótesis empleadas fueron bivariadas (correlaciones, análisis de varianza) y multivariadas (análisis MLG multivariante que proporciona un análisis de varianza para múltiples variables dependientes). De este modo, si una prueba multivariada resultaba significativa, se procedía a emplear los contrastes univariados. En caso contrario, no se continuaba con los análisis. Esto redujo la probabilidad de errores debido a la realización de múltiples comparaciones. Posteriormente, para los análisis bivariados se emplearon pruebas post hoc (i.e., Scheffé, Duncan) para determinar diferencias entre grupos.

\section{Resultados}

En la Tabla 2 se presentan los resultados obtenidos en cuanto a importancia, utilización y valoración de la calidad de vida. Se empleó el estadístico F para valorar las posibles diferencias existentes entre valoraciones, lo que nos permite afirmar que los juicios sobre importancia, utilización y valoración de la calidad de vida son distintos en las ocho dimensiones evaluadas.

Por otro lado, en cuanto a los resultados obtenidos en función de la severidad de la discapacidad, en la Tabla 3 se exponen los resultados de los análisis bivariados. Además, los análisis multivariados sobre importancia relevaron la existencia de diferencias significativas en función de esta variable (lambda de Wilks $=0.776 ; g l=24 ; p=0.0001$ ). En segundo lugar, los análisis multivariados sobre utilización evidenciaron también diferencias significativas (lambda de Wilks $=0.85 ; g l=24$; $p=0.007$ ). En la Tabla 3, se presentan los resultados obtenidos tras los análisis bivariados. En tercer lugar y en la misma línea, los análisis multivariados sobre valoración arrojaron diferencias significativas (lambda de Wilks $=0.716 ; g l=24 ; p=0.0001$ ).

TABLA 2

Puntuaciones medias en las dimensiones de la escala de Calidad de Vida

\begin{tabular}{|c|c|c|c|c|c|c|}
\hline & \multicolumn{2}{|c|}{ Importancia } & \multicolumn{2}{|c|}{ Utilización } & \multicolumn{2}{|c|}{ Valoración } \\
\hline & Media & $\mathrm{DE}$ & Media & $D E$ & Media & $D E$ \\
\hline Bienestar Emocional & 10.78 & 1.69 & 9.99 & 1.96 & 9.01 & 2.37 \\
\hline Relaciones Interpersonales & 10.67 & 1.93 & 9.53 & 2.14 & 8.64 & 2.78 \\
\hline Bienestar Material & 9.29 & 2.66 & 8.58 & 2.71 & 7.53 & 3.14 \\
\hline Desarrollo Personal & 9.46 & 2.65 & 8.95 & 2.58 & 7.48 & 2.95 \\
\hline Bienestar Físico & 14.4 & 2.41 & 13.89 & 2.34 & 12.08 & 3.14 \\
\hline Autodeterminación & 10.15 & 2.39 & 9 & 2.54 & 7.84 & 2.89 \\
\hline Inclusión Social & 9.86 & 2.51 & 8.95 & 2.61 & 7.51 & 3.02 \\
\hline Derechos & 7.06 & 1.52 & 6.58 & 1.55 & 5.13 & 2.12 \\
\hline
\end{tabular}

Fuente: elaboración propia. 
TABLA 3

Estadísticos descriptivos y significación de las diferencias entre grupos en las dimensiones de calidad de vida, en función del grado de discapacidad (ANOVA)

\begin{tabular}{|c|c|c|c|c|c|c|c|c|c|c|}
\hline \multirow[b]{2}{*}{ Variables } & \multirow[b]{2}{*}{$\mathrm{N}$} & \multicolumn{3}{|c|}{ Importancia } & \multicolumn{3}{|c|}{ Utilización } & \multicolumn{3}{|c|}{ Valoración } \\
\hline & & M & $D E$ & $F$ & $M$ & $D E$ & $\mathrm{~F}$ & $M$ & $D E$ & $F$ \\
\hline $\mathrm{BE}$ & & & & 1.609 & & & $4.812 * *$ & & & $5.295 * *$ \\
\hline Leve & 28 & 10.64 & 1.62 & & 9.64 & 1.64 & & 9.43 & 1.73 & \\
\hline Moderado & 51 & 10.53 & 1.81 & & 9.86 & 1.69 & & 9.24 & 1.93 & \\
\hline Severo & 156 & 10.97 & 1.68 & & 10.32 & 1.90 & & 9.21 & 2.33 & \\
\hline Profundo & 46 & 10.48 & 1.62 & & 9.15 & 2.36 & & 7.76 & 2.92 & \\
\hline RI & & & & $5.508 * *$ & & & $3.507^{*}$ & & & $13.954^{* * *}$ \\
\hline Leve & 28 & 11.29 & 1.08 & & 9.57 & 1.62 & & 9.86 & 1.74 & \\
\hline Moderado & 51 & 10.73 & 2.06 & & 9.29 & 1.85 & & 9.71 & 2.15 & \\
\hline Severo & 157 & 10.83 & 1.90 & & 9.83 & 2.18 & & 8.66 & 2.68 & \\
\hline Profundo & 46 & 9.70 & 2.01 & & 8.72 & 2.43 & & 6.63 & 3.20 & \\
\hline BM & & & & $11.932 * *$ & & & $7.560 * *$ & & & $17.471^{* * *}$ \\
\hline Leve & 28 & 10.32 & 1.68 & & 9.14 & 2.17 & & 9.64 & 2.23 & \\
\hline Moderado & 51 & 9.25 & 2.48 & & 8.33 & 2.38 & & 8.20 & 2.43 & \\
\hline Severo & 157 & 9.68 & 2.71 & & 9.01 & 2.83 & & 7.66 & 3.12 & \\
\hline Profundo & 46 & 7.35 & 2.29 & & 7.00 & 2.39 & & 5.02 & 2.91 & \\
\hline $\mathrm{DP}$ & & & & $3.927 * *$ & & & $4.594 * *$ & & & $8.066 * *$ \\
\hline Leve & 28 & 9.93 & 2.12 & & 9.14 & 2.16 & & 8.86 & 2.72 & \\
\hline Moderado & 51 & 9.37 & 2.31 & & 9.20 & 1.93 & & 8.24 & 2.32 & \\
\hline Severo & 157 & 9.74 & 2.82 & & 9.19 & 2.75 & & 7.43 & 2.94 & \\
\hline Profundo & 46 & 8.30 & 2.44 & & 7.67 & 2.53 & & 5.91 & 3.10 & \\
\hline Bienestar BF & & & & 2.091 & & & 1.810 & & & $6.458 * *$ \\
\hline Leve & 28 & 14.82 & 1.63 & & 14.21 & 1.87 & & 13.07 & 2.11 & \\
\hline moderado & 51 & 14.31 & 2.72 & & 14.02 & 2.14 & & 12.73 & 2.59 & \\
\hline severo & 157 & 14.58 & 2.45 & & 14.01 & 2.52 & & 12.18 & 3.24 & \\
\hline profundo & 46 & 13.65 & 2.21 & & 13.17 & 2.10 & & 10.39 & 3.38 & \\
\hline $\mathrm{AD}$ & & & & $5.870 * *$ & & & $4.138^{* *}$ & & & $16.009 * *$ \\
\hline Leve & 28 & 10.86 & 1.53 & & 9.43 & 2.04 & & 9.86 & 1.94 & \\
\hline moderado & 51 & 10.22 & 2.43 & & 8.88 & 2.08 & & 8.71 & 2.26 & \\
\hline severo & 157 & 10.36 & 2.38 & & 9.27 & 2.67 & & 7.77 & 2.91 & \\
\hline profundo & 45 & 8.87 & 2.42 & & 7.84 & 2.59 & & 5.78 & 2.65 & \\
\hline IS & & & & $6.547^{* *}$ & & & $4.133^{* *}$ & & & $12.911^{* * *}$ \\
\hline Leve & 28 & 10.39 & 1.97 & & 9.36 & 2.51 & & 9.00 & 2.46 & \\
\hline moderado & 51 & 9.69 & 2.70 & & 8.86 & 2.39 & & 8.47 & 2.29 & \\
\hline severo & 157 & 10.22 & 2.42 & & 9.25 & 2.63 & & 7.52 & 2.98 & \\
\hline profundo & 46 & 8.48 & 2.49 & & 7.78 & 2.57 & & 5.41 & 3.08 & \\
\hline $\mathrm{DE}$ & & & & $4.007 * *$ & & & 1.900 & & & $6.508^{* *}$ \\
\hline Leve & 28 & 7.39 & 1.40 & & 6.71 & 1.65 & & 6.00 & 1.94 & \\
\hline moderado & 51 & 7.04 & 1.67 & & 6.73 & 1.40 & & 5.43 & 1.98 & \\
\hline severo & 157 & 7.20 & 1.47 & & 6.66 & 1.60 & & 5.20 & 2.13 & \\
\hline profundo & 46 & 6.39 & 1.44 & & 6.09 & 1.46 & & 4.02 & 1.99 & \\
\hline
\end{tabular}

Nota. ${ }^{*}$ p $<$ 0.01. BE = Bienestar Emocional; RI = Relaciones Interpersonales; BM = Bienestar Material; DP = Desarrollo

Personal; $\mathrm{BF}=$ Bienestar Físico; $\mathrm{AD}=$ Autodeterminación; $\mathrm{IS}=$ Inclusión Social; $\mathrm{DE}=$ Derechos.

Fuente: elaboración propia. 


\section{TABLA 4}

Estadísticos descriptivos y significación de las diferencias entre grupos en importancia de dimensiones de calidad de vida, en función de los años de institucionalización (ANOVA)

\begin{tabular}{|c|c|c|c|c|c|}
\hline & $N$ & Media & Desviación Estandar & $F$ & Sig. \\
\hline $\mathrm{BE}$ & & & & 5.546 & 0.004 \\
\hline Hasta 12 & 131 & 10.72 & 1.83 & & \\
\hline De 13 a 24 & 92 & 11.18 & 1.26 & & \\
\hline 25 o más & 59 & 10.27 & 1.85 & & \\
\hline RI & & & & 2.381 & 0.094 \\
\hline Hasta 12 & 132 & 10.58 & 2.09 & & \\
\hline De 13 a 24 & 92 & 11.01 & 1.53 & & \\
\hline 25 o más & 59 & 10.36 & 2.04 & & \\
\hline BM & & & & 3.179 & 0.043 \\
\hline Hasta 12 & 132 & 9.15 & 2.72 & & \\
\hline De 13 a 24 & 92 & 9.83 & 2.48 & & \\
\hline 25 o más & 59 & 8.78 & 2.70 & & \\
\hline $\mathrm{DP}$ & & & & 7.276 & 0.001 \\
\hline Hasta 12 & 132 & 9.48 & 2.67 & & \\
\hline De 13 a 24 & 92 & 10.09 & 2.22 & & \\
\hline 25 o más & 59 & 8.44 & 2.93 & & \\
\hline $\mathrm{BF}$ & & & & 1.526 & 0.219 \\
\hline Hasta 12 & 132 & 14.30 & 2.65 & & \\
\hline De 13 a 24 & 92 & 14.74 & 1.92 & & \\
\hline 25 o más & 59 & 14.08 & 2.51 & & \\
\hline $\mathrm{AD}$ & & & & 3.135 & 0.045 \\
\hline Hasta 12 & 132 & 10.04 & 2.51 & & \\
\hline De 13 a 24 & 92 & 10.62 & 1.86 & & \\
\hline 25 o más & 58 & 9.67 & 2.73 & & \\
\hline IS & & & & 1.611 & 0.202 \\
\hline Hasta 12 & 132 & 9.77 & 2.68 & & \\
\hline De 13 a 24 & 92 & 10.22 & 2.13 & & \\
\hline 25 o más & 59 & 9.51 & 2.63 & & \\
\hline $\mathrm{DE}$ & & & & 0.518 & 0.596 \\
\hline Hasta 12 & 132 & 7.01 & 1.62 & & \\
\hline De 13 a 24 & 92 & 7.20 & 1.40 & & \\
\hline 25 o más & 59 & 6.98 & 1.48 & & \\
\hline
\end{tabular}

Nota BE $=$ Bienestar Emocional; $\mathrm{RI}=$ Relaciones Interpersonales; $\mathrm{BM}=$ Bienestar Material; $\mathrm{DP}=$ Desarrollo Personal; $\mathrm{BF}=$ Bienestar Físico; $\mathrm{AD}=$ Autodeterminación; IS = Inclusión Social; $\mathrm{DE}=$ Derechos

Fuente: elaboración propia.

En la mencionada Tabla 3, se puede observar cómo las personas con una discapacidad intelectual profunda obtienen una valoración significativamente inferior en todas las dimensiones. Estos resultados fueron confirmados por los análisis post hoc (Scheffé y Duncan) de diferencias entre grupos.
Respecto al impacto de la institucionalización, los contrastes multivariados sobre importancia resultaron significativos (lambda de Wilks = 0.899; $g l=16 ; p=0.023)$. En la Tabla 4, se presentan los resultados obtenidos. Se puede observar cómo el grupo institucionalizado más de 25 años obtuvo puntuaciones significativamente inferiores en Bien- 
estar Emocional, Bienestar Material, Desarrollo Personal y Autodeterminación. Por otro lado, los contrastes multivariados sobre utilización pusieron de manifiesto la ausencia de diferencias significativas entre grupos (lambda de Wilks $=0.924 ; g l=16$; $p=0.151)$. Tampoco lo fueron los contrastes multivariados sobre valoraciones de calidad de vida (lambda de Wilks $=0.937 ; g l=16 ; p=0.343$ ).

\section{Discusión}

Los resultados del presente estudio permiten acumular evidencias sobre la adecuación de la Escala Transcultural de Calidad de Vida para evaluar, en el contexto chileno, la situación de personas con discapacidad intelectual institucionalizadas respecto de su calidad de vida. Estos resultados complementan los hallazgos de trabajos previos (Jenaro et al., 2005; Schalock et al., 2005). Permiten además proponer el uso de esta herramienta como estrategia para evaluar y reflexionar sobre los servicios que se ofrecen a este colectivo u otros similares en situación de desventaja. En definitiva, su empleo reorienta los servicios hacia la mejora de la calidad de vida (Schalock \& Verdugo, 2003; AAIDD, 2010).

Los resultados posibilitan también extraer reflexiones sobre el enfoque que se adopta en la provisión de servicios para personas con discapacidad. Como se indicó antes, unos servicios centrados en el cliente debieran caracterizarse por la elevada importancia y utilización de las diversas dimensiones e indicadores de calidad de vida. Esto debería plasmarse, a su vez, en una vida de calidad. En este sentido, las correlaciones obtenidas en el presente estudio son ligeramente inferiores a las halladas en trabajos previos (Jenaro et al., 2005; Schalock et al., 2005). Lo anterior puede deberse al hecho de que si bien en Chile el concepto de calidad de vida se considera un principio esencial en este colectivo (i. e., importante), aún no se ha plasmado en las prácticas (i. e., utilización), especialmente en un colectivo como el que nos ocupa y que se caracteriza por su situación de exclusión. En apoyo de esta explicación es posible atender a las puntuaciones promedio obtenidas en las dimensiones de calidad de vida (véase Tabla 2), donde se aprecia que las puntuaciones más elevadas se otorgan a las dimensiones de Bienestar Físico y Emocional, mientras que las más bajas a la dimensión de Derechos. Esto sugiere la necesidad de seguir trabajando en la mejora de los servicios, para pasar de un enfoque centrado en la satisfacción de necesidades básicas a otro caracterizado por la defensa de los plenos derechos de esta población (Gomez et al., 2010).

De hecho, el hallazgo de la existencia de diferencias en cuanto a los juicios sobre importancia, utilización y valoración de la calidad de vida sugieren que si bien esta última noción está siendo trabajada como principio importante en los servicios para personas con discapacidad, aún no está plenamente incorporada y, sobre todo, sus efectos no se están percibiendo en los destinatarios de estos servicios. Estos resultados remarcan la necesidad de ir trabajando en pro de la desinstitucionalización y hacia una vida en comunidad, teniendo en cuenta las mejoras que ello acarrea en el bienestar experimentado por esta población (Chou \& Schalock, 2007; Felce, 1998; Mansell, 2006; Mansell \& Beadle-Brown, 2010).

Los resultados obtenidos evidencian que, de acuerdo a las predicciones de este estudio, las personas con discapacidad leve o moderada presentan una mejor calidad de vida en cuanto a importancia, uso y valoración. Esto concuerda con numerosas investigaciones que indican que el grado de funcionamiento está relacionado con la calidad de vida (Borthwick-Duffy, 1992; Jenaro et al., 2005; Ouellette-Kuntz \& McCreary, 1996; Schalock et al., 2005). Esto nos lleva a resaltar la importancia de promover una vida de calidad especialmente en un colectivo de riesgo como son quienes presentan más necesidades de apoyo. Un servicio centrado en la persona y en la mejora de su calidad de vida debe actuar (utilización) en consonancia con la relevancia (importancia) otorgada a las diferentes dimensiones de calidad de vida, lo que a su vez se debe reflejar en el bienestar (valoración) experimentado por la persona con discapacidad.

Un tema que sin duda requiere de más estudios es el relativo al impacto de la institucionalización. El hecho de que se conceda una menor importancia al Bienestar Emocional, Bienestar Material, Desa- 
rrollo Personal y Autodeterminación de quienes llevan más tiempo institucionalizados debe llevar a reflexionar sobre el impacto negativo de una prolongada institucionalización, como de hecho se ha constatado en otros estudios (Felce \& Emerson, 2001; Stancliffe, 2002; Stancliffe \& Parmenter, 1999; Stancliffe et al., 2002; Young, 2006), de ahí la importancia de poner en marcha actuaciones en la población institucionalizada, que potencien sus relaciones con otras personas, sus redes sociales y su participación en la comunidad (Heller, 2002; Verdonschot, Witte, Reicharth, Buntix \& Curf, 2009).

Los resultados destacan la necesidad de reorientar las prácticas en los centros residenciales, desplazándose desde un modelo de "cuidados totales" hacia otro basado en una "vida con apoyo". Si bien las instituciones cumplen un papel clave para garantizar la protección de sus residentes, es preciso seguir avanzando en pro de una plena inclusión social y autodeterminación (Cruz, Pérez, Jenaro, Vega $\&$ Flores, 2011; Vega et al., 2011). Y esto porque, según demuestran estudios previos, la calidad de vida está condicionada por las oportunidades de inclusión social que se ofrezcan (Córdoba, Mora, Bedoya \& Verdugo, 2007). En definitiva, gozar de una elevada calidad de vida no ha de ser privativo de un determinado grupo de población, sino que ha de constituir una meta a la que toda persona, con o sin discapacidad, debe aspirar.

No queremos finalizar este trabajo sin aludir a varias limitaciones que habrán de ser solventadas en posteriores estudios. En primer lugar, el estudio ha empleado una muestra de conveniencia perteneciente a una única fundación chilena. Si bien esta es la más importante de Chile, es necesario confirmar los resultados obtenidos con otras poblaciones pertenecientes a otras instituciones que, en el momento de realización de este estudio, declinaron participar. Por otro lado, se ha de destacar que la evaluación ha sido llevada a cabo por informantes, en coherencia con el objetivo de verificar la aplicabilidad de la Escala Transcultural de Calidad de Vida. Sin embargo, posteriores estudios debieran centrarse en la valoración de la calidad de vida informada por las propias personas con discapacidad. Del mismo modo, sería de utilidad emplear instrumentos adi- cionales para evaluar las necesidades de apoyo de este colectivo y su impacto en la calidad de vida, aprovechando la existencia de escalas novedosas y con adecuadas propiedades psicométricas como la Escala de Intensidad de Apoyos SIS (Verdugo, Arias \& Ibáñez, 2007). De modo adicional, el empleo de metodologías cualitativas permitiría profundizar en las percepciones de profesionales y usuarios sobre estos aspectos, así como sobre factores asociados. Pese a las limitaciones comentadas, el estudio arroja luz sobre una realidad aún poco estudiada y que requiere de esfuerzos de evaluación, intervención y de mejora de servicios y prácticas.

\section{Referencias}

Albert, S. M. (1997). Assessing health-related quality of life in chronic care populations. Journal of Mental Health and Aging, 3(1), 101-118.

American Association on Intellectual and Developmental Disabilities. (2010). Intellectual disability. Definition, classification and systems of support. Washington, DC: Autor.

Anderson, R., Aaronson, N. \& Wilkin, D. (1993). Critical review of the international assessments of health-related quality of life. Quality of Life Research, 2(6), 369-395.

Annison, J. (2000). Towards a clearer understanding of the meaning of "home". Journal of Intellectual $\mathbb{E}$ Developmental Disability, 25(4), 251-262.

Borthwick-Duffy, S. (1992). Quality of life: The residential environmental. En W. E. Kierman \& R. L. Schalock (Eds.), Economics, industry, and disability: A look ahead (pp. 351-363). Maryland: Paul H. Brookes.

Brown, R. I. (1988). Quality of life for people with disabilities. Models research ad practice (2a. ed.). Cheltenhan, UK: Stanley Thornes.

Brown, R. \& Brown, I. (2005).The application of quality of life. Journal of Intellectual Disability Research, 49(10), 718-727.

Chou, Y. \& Schalock, R. (2007). Trends in residential policies and services for people with intellectual disabilities in Taiwan. Journal of Intellectual Disability Research, 51(2), 135-141. 
Córdoba, L., Mora, A., Bedoya, A. \& Verdugo, M. A. (2007). Families of adults with intellectual disability in Cali, Colombia, using the model Quality of Life. Psykhe, 16(2), 29-42.

Cruz, M., Pérez, M. C., Jenaro, C., Vega, V. \& Flores, N. (2011). Vivenciando las necesidades de apoyo en la enfermedad mental. Index de Enfermería, 20(1-2), 16-20.

Cummins, R. A. (2005). Moving from the quality of life concept to a theory. Journal of Intellectual Disability Research, 49(10), 699-706.

Felce, D. (1998). The determinants of staff and resident activity in residential services for people with severe intellectual disability: Moving beyond size, building design, location and number of staff. Journal of Intellectual 8 Developmental Disability, 23(2), 103-119.

Felce, D. \& Emerson, E. (2001). Living with support in a home in the community: Predictors of behavioral development and household and community activity. Mental Retardation and Developmental Disabilities Research Reviews, 7(2), 75-83.

Felce, D. \& Perry, J. (1995). Quality of life: Its definition and measurement. Research in Developmental Disabilities, 16(1), 51-74.

Goode, D. A. (1989). Quality of life and quality of work life. En W. E. Kiernan \& R. L. Schalock (Eds.), Economics industry and disability: A look ahead (pp. 337-349). Baltimore, Maryland: Paul H. Brookes.

Garin, O., Ferrer, M., Pont, A., Rue, M., Kotzeva, A., Wiklund, I., et al. (2009). Disease-specific healthrelated quality of life questionnaires for heart failure: A systematic review with meta-analyses. Quality of Life Research, 18(1), 71-85.

Gomez, L. E., Verdugo, M. A. \& Arias, B. (2010). Individual quality of life: Advances in the conceptualization, and emerging challenges in the disability field. Behavioral Psychology/Psicologia Conductual, 18(3), 453-472.

Hatton, C., Emerson, E., Robertson, J., Henderson, D. \& Cooper, J. (1995). The quality and costs of residential services for adults with multiple disabilities: A comparative evaluation. Research in Developmental Disabilities, 16(6), 439-460.
Heller, T. (2002). Residential settings and outcomes for individuals with intellectual disabilities. Current Opinion in Psychiatry, 15(5), 503-508.

Jenaro, C., Verdugo, M. A., Caballo, C., Balboni, G., Lachapelle, Y., Otrebski, W., et al. (2005). Crosscultural study of person-centred quality of life domains and indicators: A replication. Journal of Intellectual Disability Research, 49(10), 734-739.

Keith, R. \& Rawlinson, R. (1998). Quality of life issues in the development and evaluation of services for people with intellectual disability. Journal of Intellectual and Developmental Disability, 23(3), 199-218.

Mansell, J. (2006). Deinstitutionalisation and community living: Progress, problems and Priorities. Journal of Intellectual and Developmental Disability, 31(2), 65-76.

Mansell, J. \& Beadle-Brown, J. (2010). Deinstitutionalization and community in living: Position statement of the comparative policy and practice special interest research group of the International Association for the Scientific Study of Intellectual Disabilities. Journal of Intellectual Disability Research, 54(2), 104-112.

Murdaugh, C. (1997). Health-related quality of life as an outcome in organizational research. Medical Care, 35(Suppl. 11), NS41-NS48.

Murphy, S. T., Rogan, P. M., Handley, M., Kincaid, C. $\&$ Royce-Davis, J. (2002). People's situations and perspectives eight years after workshop conversion. Mental Retardation, 40(1), 30-40.

Naughton, M. \& Wiklund, I. (1993). A critical review of dimension-specific measures of health-related quality of life in cross-cultural research. Quality of Life Research, 2(6), 397-432.

Ouellette-Kuntz, H. \& McCreary, B. D. (1996). Quality of life assessment for persons with severe developmental disabilities. En R. Renwick, I. Brown \& M. Nagler (Eds.), Quality of life in health promotion and rehabilitation: Conceptual approaches, issues, and applications (pp. 268-278). Thousand Oaks, CA: Sage.

Palfreyman, S. J., Tod, A. M., Brazier, J. E. \& Michaels, J. A. (2010). A systematic review of health-related quality of life instruments used for people with venous ulcers: An assessment of their suitability 
and psychometric properties. Journal of Clinical Nursing, 19(19-20), 2673-2703.

Quittner, A. L., Cruz, I., Modi, A. C. \& Marciel, K. K. (2009). Health-related quality of life instruments for adolescents with chronic diseases. En W. O'Donohue \& L. Tolle (Eds.), Behavioral approaches to chronic disease in adolescence: A guide to integrative care (pp. 311-327). New York: Springer.

Revicki, D. \& Kaplan, R. (1993). Relationship between psychometric and utility-based approaches to the measurement of health-related quality of life. Quality of Life Research, 2(6), 477-487.

Schalock, R. L., Bonham, G. \& Verdugo, M. A. (2008). The conceptualization and measurement of quality of life: Implications for program planning and evaluation in the field of intellectual disabilities. Evaluation $\mathcal{E}$ Program Planning, 31(2), 181-190.

Schalock, R. L., Gardner, J. F. \& Bradley, V. J. (2007). Quality of life for people with intellectual and other developmental disabilities: Applications across individuals, organizations, communities, and systems. Washington, DC: American Association on Intellectual and Developmental Disabilities.

Schalock, R. \& Verdugo, M. A. (2003). Calidad de Vida. Manual para profesionales de la educación, salud y servicios sociales. Madrid: Alianza.

Schalock, R., Verdugo, M. A., Jenaro, C., Wang, M., Wehmeyer, M., Jiancheng, X., et al. (2005). Crosscultural study of quality of life indicators. American Journal on Mental Retardation, 110(4), 298-311.

Schalock, R. L., Keith, K. D., Verdugo, M. A. \& Gomez, L. E. (2010). Quality of life model development in the field of intellectual disability. En R. Kober (Ed.), Enhancing quality of life for people with intellectual disability: From theory to practice (pp. 17-32). New York: Springer.

Solans, M., Pane, S., Estrada, M. -D., Serra-Sutton, V., Berra, S., Herdman, M., et al. (2008). Healthrelated quality of life measurement in children and adolescents: A systematic review of generic and disease-specific instruments. Value in Health, 11(4), $742-764$.
Stancliffe, R. (2002). Provision of residential services for people with intellectual disability in Australia: An international comparison. Journal of Intellectual \& Developmental Disability, 27(2), 117-124.

Stancliffe, R. J., Hayden, M. F., Larson, S. \& Lakin, K. C. (2002). Longitudinal study on the adaptive and challenging behaviors of deinstitutionalized adults with intellectual disability. American Journal on Mental Retardation, 107(4), 302-320.

Stancliffe, R. \& Parmenter, T. R. (1999). The Choice Questionnaire: A scale to assess choices exercised by adults with intellectual disability. Journal of Intellectual and Developmental Disability, 24(2), 107-132.

Thompson, J., Bradley, V., Buntinx, W., Schalock, R., Shogren, K., Snell, M., et al. (2009). Conceptualizing supports and the support needs of people with intellectual disability. Intellectual and Developmental Disabilities, 4(2), 135-146.

Vega, V., Jenaro, C., Cruz, M. \& Flores, N. (2011). Calidad de vida de adultos con discapacidad intelectual en centros residenciales permanentes chilenos. Siglo Cero, 42(2), 26-38.

Verdonschot, M. M., Witte, L. P., Reichrath, E., Buntinx, W. H. \& Curfs, L. M. (2009). Impact of environmental factors on community participation of persons with an intellectual disability: A systematic review. Journal of Intellectual Disability Research, 53(1), 54-64.

Verdugo, M. A., Arias, B. \& Ibáñez, A. (2007). SIS. Escala de Intensidad de Apoyos. Madrid: TEA.

Verdugo, M. A., Schalock, R., Wehmeyer, M., Caballo, C. \& Jenaro, C. (2001). Indicadores transculturales de calidad de vida: evaluación de su importancia y uso. Salamanca: Universidad de Salamanca.

Young, L. (2006). Community and cluster centre residential services for adults with intellectual disability: Long-term results from an Australian-matched sample. Journal of Intellectual Disabilities, 50(6), 419-431. 
